\title{
Cis-platinum chemotherapy for ocular basal cell carcinoma
}

\author{
Michael Morley, Paul T Finger, Martin Perlin, Lora R Weiselberg, Daniel S DeBlasio
}

\begin{abstract}
We have used intravenous cis-platinum chemotherapy in the treatment of three patients with basal cell carcinoma of the lid extending into the orbit. Cis-platinum chemotherapy caused a reduction in tumour size and thereby delayed surgery in all cases. It allowed for local resection in one case, appeared to delay a patient's exenteration in a second case, and was used prior to radiotherapy in a third case. While not curative, cis-platinum may be useful as an adjuvant to decrease tumour mass prior to local excision and for patients who refuse or must delay exenteration.
\end{abstract}

Basal cell carcinoma is the commonest malignant tumour of the eyelids. ${ }^{1}$ Surgical excision is the treatment of choice because of the high rate of cure and excellent cosmetic and functional results. ${ }^{2-14}$ However, there are circumstances when surgical resection is impossible or when a patient refuses to have surgery. Other forms of treatment have been described including radiotherapy, chemotherapy, phototherapy, drug therapy, immunotherapy, and cryotherapy. ${ }^{15-24}$ Radiotherapy has been successful in the treatment of basal cell carcinoma, but associated lash loss, dry eye, keratopathy, cataract, retinopathy, and optic neuropathy have been reported. ${ }^{15} 16$ While cryotherapy has been used for small superficial tumours, newer methods including immunotherapy, phototherapy, and drug therapy are still being investigated. ${ }^{20-25}$

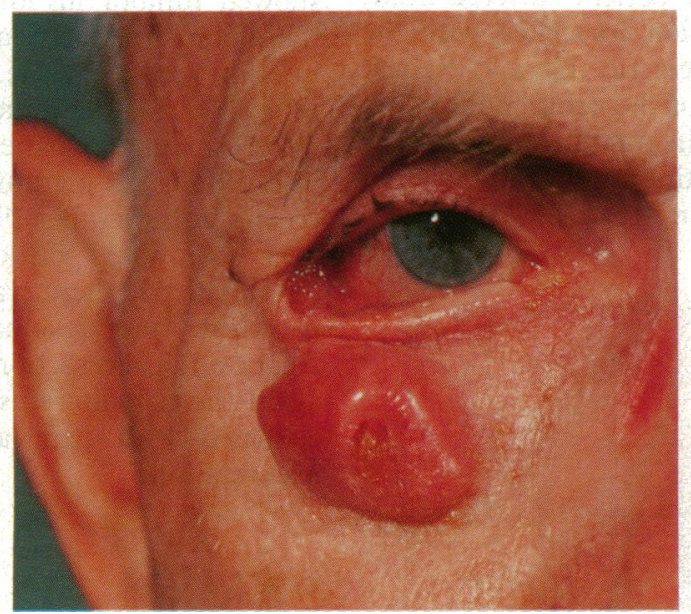

Figure $1 A$

Figure 1(A) A pretreatment external photograph of the nodular basal cell carcinoma on the right lower lid (15 August 1985). Note the tumour-associated lateral ectropion. (B) $A$ post-treatment external photograph of the nodular basal cell carcinoma on the right lower lid two months after the onset of intravenous cis-platinum chemotherapy (15 October 1985).

Note the marked reduction in size of the superficial tumour and partial resolution of the lateral ectropion.

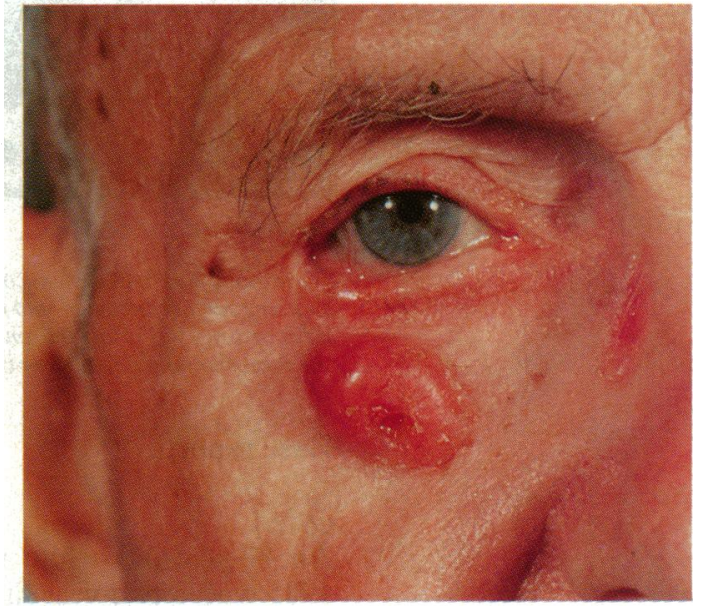

Figure $1 B$

Chemotherapy of basal cell carcinoma has also been tried as treatment for tumours involving the orbit and adnexa. ${ }^{17-19} \mathrm{~A}$ total of eight cases of chemotherapy as a primary treatment for basal cell carcinoma in the eyelids have been reported. ${ }^{1718}$ We describe our experience in treating three cases of basal cell carcinoma involving the orbit and eyelids with intravenous cis-platinum.

\section{Materials and methods}

Three patients with biopsy proved basal cell carcinoma and who refused exenteration were offered chemotherapy as a possible alternative to surgery. Our treatment protocol was a variation of that described by Luxenberg and Guthrie. ${ }^{17} 18$ The patients were admitted to hospital and followed up by an ophthalmologist and medical oncologist. On admission the height and weight were measured to calculate body surface area (BSA). Routine admission laboratory studies included creatinine clearance, sequential multiple analyser profile-20, prothrombin time, activated partial thromboplastin time, electrocardiogram, chest $x$ ray, complete blood count, and urine analysis. Patients were hydrated with normal saline, $125 \mathrm{ml} / \mathrm{h}$, overnight prior to chemotherapy. On the day of chemotherapy, $20 \%$ mannitol $(100 \mathrm{ml})$ was administered intravenously, followed immediately by cis-platinum (75-85 $\mathrm{mg} / \mathrm{square}$ metre BSA) run in over two hours. Vigorous hydration was continued for 24 hours. Dexamethasone phosphate $10 \mathrm{mg}$, metoclopramide $50 \mathrm{mg}$, diphenhydramine hydrochloride $50 \mathrm{mg}$, perchlorperazine edisylate 10 $\mathrm{mg}$, and droperidol $5 \mathrm{mg}$ were used to reduce the side effects of the cis-platinum. Fluid balance, electrolytes, and renal function were monitored after administration of chemotherapy. Patients were discharged within 48 hours after chemotherapy. 


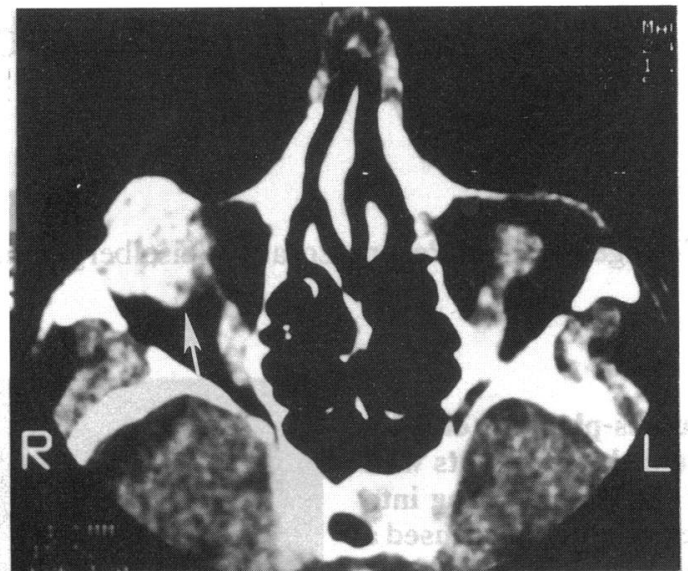

Figure 2A

Figure 2(A) Pretreatment computed tomography of the orbit showing tumour invasion into the inferior and inferotemporal orbit (arrow). (B) Post-treatment computed tomography of the orbit showing a reduction in tumour volume (arrow).

\section{Case reports}

CASE 1

An 80-year-old white male presented in May 1985 with a two-year history of a right lower lid mass (Figs 1A). Ophthalmic examination revealed 20/40 vision in the right eye and full ocular motility. Slit-lamp biomicroscopy revealed a lateral ectropion and an extensive nodular tumour in the right lower lid. Computed tomography of the orbit demonstrated a mass on the right lower lid, extending into the inferior orbit to the level of the inferior rectus (Figs 2A). An incisional biopsy revealed basal cell carcinoma. Our patient refused surgical resection and was offered the alternative of cis-platinun chemotherapy.

Cis-platinum was administered without complication in July 1985 . Two weeks later the tumour had regressed in size by approximately $30 \%$ (Fig 1B). A second course of cis-platinum was given in August 1985, and two weeks later the tumour was reduced in size by approximately $40 \%$. We noted that the patient suffered mild, transient mental confusion associated with the chemotherapy and antiemetic therapy. Repeat computed tomography of the orbit confirmed a reduction in the size of the tumour (Fig 2B), and a third course of cis-platinum was administered in October 1985. Again treatment was com-

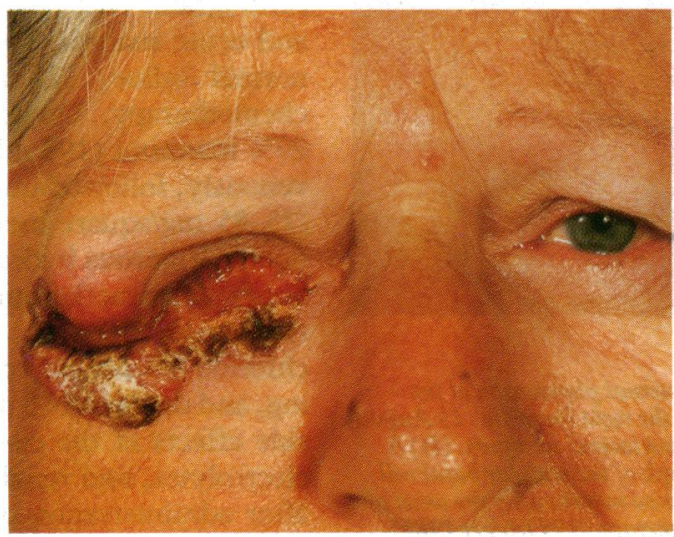

Figure 3 Large mass extending from the medial canthus across the entire lower lid to $5 \mathrm{~mm}$ beyond the lateral canthus.

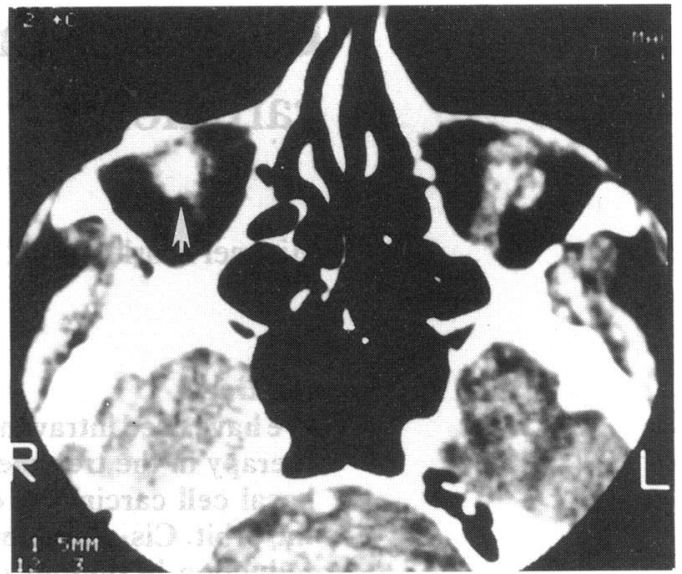

Figure 2B

plicated by transient night time mental confusion, this time requiring treatment with haloperidol. Because of the difficulty with therapy and the reduction in the size of the tumour and its intraorbital extent, this patient elected to proceed with local surgical excision in November 1985. An anterior orbitotomy and tumour excision with frozen section control provided negative margins. Then a modified semicircular flap with skin grafting was performed. Postoperative examinations showed no loss of vision and normal ocular motility. Permanent margins were negative for carcinoma. Follow-up reports four years later showed no evidence of recurrence.

CASE 2

A 66-year-old white female presented in August 1985 with a five-year history of a mass on the right lower lid. Ophthalmic examination showed a visual acuity of $20 / 20$ in the right eye with restriction of infraduction. A large mass extended from the medial canthus across the entire lower lid to $5 \mathrm{~mm}$ beyond the lateral canthus (Fig 3). An incisional biopsy revealed basal cell carcinoma. Computed tomography of the orbit showed tumour invading the orbit, displacing the globe superiorly, and contiguous with the inferior rectus muscle. Surgical resection was refused. Cis-platinum chemotherapy was offered, and the patient received a course of intravenous cis-platinum in September and a second in November 1985. While partial regression was noted on clinical and repeat CT examinations, there was still obvious lid, conjunctival, and residual orbital involvement. Again surgical resection was recommended, and again the patient refused. In August 1986 she returned with pain and agreed to undergo exenteration.

\section{CASE 3}

A 68-year-old white male presented in December 1983 with a right lower lid mass present for one year. The lesion was noted to involve a $33 \mathrm{~mm}$ of the lower lid, and was diagnosed as an infiltrating morpheaform basal cell carcinoma by incisional biopsy. Sinus $x$ rays and CT appearances of the orbit and sinuses were normal. He was treated with electron beam (external) radiation therapy 
and was irradiated with $5977 \mathrm{cGy}$ in 30 fractions over 44 days in March 1984. The tumour appeared to respond well to the treatment, with complete regression of the visible part. Post irradiation epiphora and cicatrix formation with entropion were noted.

In May 1987, after three years of local control, the patient returned with recurrent basal cell carcinoma of the right lower lid and a mucoid discharge from the medial canthal area. The visual acuity in the right eye was 20/40 and he had full ocular motility. Computed tomography of the orbit revealed an abnormal soft tissue density anterior and medial to the globe with slight postseptal extension (Fig 4A). The ethmoidal and frontal sinuses showed mucosal thickening and bony erosion, with no evidence of tumour extension. An incisional biopsy was positive for basal cell carcinoma. Surgical resection was recommended, but since the tumour appeared to be localised to the nasal orbit a presurgical trial of cis-platinum chemotherapy was offered. The patient underwent three courses of chemotherapy in August 1987, September 1987, and November 1987 with reduction in the apparent size of the mass (Fig 4B). Since he developed severe akathisia after receiving metachlopromide with his first cycle of treatment, lorazepam was subsequently used as an antiemetic agent. The patient did not return for follow-up until June 1988, and a repeat biopsy again revealed basal cell carcinoma. Two additional cycles of chemotherapy were administered in July and September 1988. Then in December the patient was noted to have a rise in his creatinine level to $168 \mu \mathrm{mol} / \mathrm{l}$ and a reduction in his creatinine clearance to $46 \mathrm{ml} / \mathrm{min}$. This precluded further chemotherapy.

We undertook extensive lid, orbit, and sinus resection in an attempt to excise the tumour. Multiple frozen sections were positive for tumour, and I-125 brachytherapy implants were placed in the frontal sinus and against the remaining ethmoidal sinuses as a $30 \mathrm{~Gy}$ boost to external radiotherapy. Then $60 \mathrm{~Gy}$ of external beam radiation was delivered to the orbit and sinuses. Eighteen months after radiotherapy there has been no evidence of new tumour growth.

\section{Discussion}

This is the second series of cases recording a reduction in the size of basal cell carcinomas of the lids and orbit associated with cis-platinum chemotherapy. All three patients we treated were considered to have partial regression of their tumour. In one of the three cases partial regression allowed for local excision with negative margins, in another delayed exenteration, and in the third it postponed radiotherapy.

There are significant side effects associated with intravenous cis-platinum chemotherapy. ${ }^{26-34}$ As seen in case 3 in our report, dose related and cumulative renal insufficiency is the major toxic effect. ${ }^{26}$ Thus pre-existing renal insufficiency is a contraindication to its use. Careful attention to fluid status, creatinine clearance, and creatinine levels is mandatory.

Severe nausea and vomiting are frequently

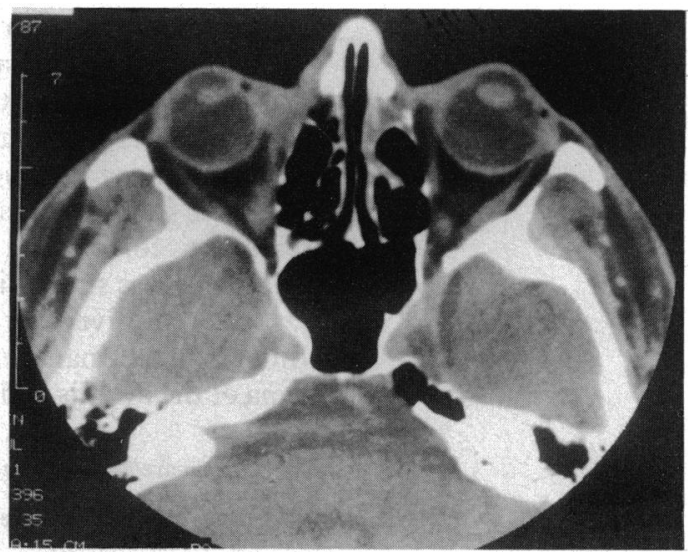

Figure 4A

Figure 4(A) Computed tomography of the orbit showing an abnormal soft-tissue density medial and anterior to the globe. Postseptal extension is noted. Bone thinning and mucosal thickening was noted in the sinuses. (B) Post-treatment computed tomography of the orbit showing a reduction in the apparent size of the tumour after three courses of cis-platinum chemotherapy.

observed after the administration of cisplatinum, so that administration of antiemetics is standard practice. Two of our patients (cases 1 and 3) had neurological reactions to the combination of medicines used in this protocol. These reactions were probably due to the antiemetic therapy, not the cis-platinum. Ototoxicity has been observed in some patients, usually manifested by tinnitus or high frequency hearing loss. ${ }^{27}$

There are a number of reports describing the ocular side effects of cis-platinum or chemotherapeutic regimens that contain cis-platinum: cortical blindness, optic disc swelling, retrobulbar neuritis, homonomous hemianopsia, retinal pigmentary changes, abnormalities of electroretinogram and visually evoked potentials and encephalopathy. ${ }^{28-34}$

While the preferred method of treatment of basal cell carcinoma is surgical excision, there are circumstances when that is impossible. Occasionally a patient refuses to have surgery. In cases where extensive surgery is refused, less extensive surgery coupled with an alternative treatment may be effective.

All three patients reported on here, including one who was pretreated with radiation therapy, had an objective response to treatment. No case attained complete remission, and all required

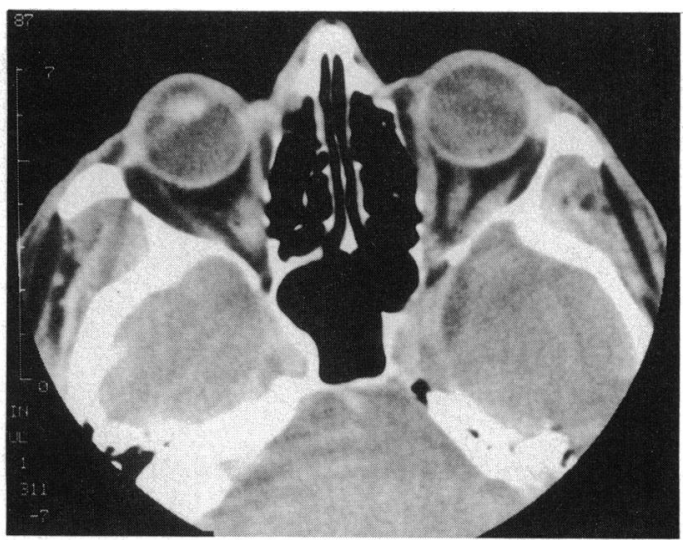

Figure $4 B$ 
further treatment. Our data confirm the observations of Luxenberg and Guthrie that lid and periocular basal cell carcinoma will respond to intravenous cis-platinum with adriamycin. ${ }^{17} 18$ their cases the commonest outcome was a partial reduction in tumour size requiring additional treatment to control tumour spread. Our experience was similar in that cis-platinum alone was not curative, yet it served as a helpful adjunct in the management of basal cell carcinoma of the eyelids and orbit.

The authors have no proprietary interest in the chemotherapeutic agent cis-platinum.

We thank Dr Frederick A Jakobiec, for his original suggestion to contact Dr Luxenburg, and Dr Jason Slakter, Dr Peter Small, Dr Jay L Bosworth, and Dr Samuel Packer for sharing in the care of these patients.

1 Reese AB. Epithelial tumors of the lid, conjunctiva, cornea, and lacrimal sac. In: Reese AB, ed. Tumors of the eye Hagerstown: Harper and Row: 1976; chapter 1: 38-62

2 Mohs FE. Chemosurgery: microscopically controlled method of cancer excision. Arch Surg 1941; 42: 279-95.

3 Hughes WL. Reconstruction of the lid. Am $f$ Ophthalmol 1945; 28 : 1203-11.

4 Cutler NL, Beard C. A method for partial and total upper eyelid reconstruction. Am $\mathcal{F}$ Ophthalmol 1955; 39: 1-7.

5 Smith B, Obear MF. Bridge flap technique for reconstruction of large upper eyelid defects. Ophthalmology 1967; 71: 897of large upper eyelid defects. Ophthalmology 1967; 71: 897 901.

6 Youens WT, Westphal C, Barfield FT Jr, Youens H. Full thickness lower lid transplant. Arch Ophthalmol 1967; 77 226-9.

7 Fox SA. Autogenous free full thickness eyelid grafts. Am $\mathcal{F}$ Ophthalmol 1969; 67: 941-5.

8 Beard C. Observations on the treatment of basal cell carcinoma of the eyelids. Ophthalmology 1975; 79: 664-70.

9 Tenzel RR. Reconstruction of the central one half of the eyelid. Arch Ophthalmol 1975; 93: 125-6.

10 Older JJ, Quickert MH, Beard C. Surgical removal of basal cell carcinoma of the eyelids utilizing frozen section control. Ophthalmology 1975; 79: 658-63.

11 Putterman AM. Viable composite grafting in eyelid reconstruction. Am $\mathcal{F}$ Ophthalmol 1978; 85: 237-41.

12 Tenzel RR, Stewart WB. Eyelid reconstruction by semicircula flap technique. Ophthalmology 1978; 85: 1164-9.

13 Anderson RL, Edwards JJ. Reconstruction by myocutaneou eyelid flaps. Arch Ophthalmol 1979; 97: 2358-62.

14 McCord CD, Wesley RE. Reconstruction of the upper eyelid and medial canthus. In: McCord CD, Jr, ed. Oculoplastic surgery. New York: Raven, 1981: 175-88.

15 Fitzpatrick PJ, Thompson GA, Esterbrook WM, Gallie BL Payne DG. Basal and squamous cell carcinoma of the evelids and their treatment by radiotherapy. Int $\mathcal{F}$ Radiat Oncol Biol Phys 1984; 10: 449-54.

16 Rodriquez-Sains RS, Perry R, Smith B, Bosniak S. Radiotherapy of periocular basal cell carcinomas: recurrence rate and treatment with special attention to the medial canthus. and treatment with special attention

17 Luxenberg MN, Guthrie TH. Chemotherapy of eyelid and periorbital tumors. Trans Am Ophthalmol Soc 1985; 83: 16280.

18 Luxenberg MN, Guthrie TH. Chemotherapy of basal cell and squamous cell carcinoma of the eyelids and periorbital tissues. Ophthalmology 1986; 93: 504-10.

19 Robinson JK. Use of combination chemotherapy and radiation therapy in the management of advanced basal cell carcinoma of the head and neck. F Am Acad Dermatol 1987; 17 (5): 7704.

20 Fraunfelder FT, Zacarian SA, Wingfield DL, Limmer BL. Results of cryotherapy for eyelid malignancies. Am $\mathcal{F}$ Ophthalmol 1984; 97: 184-8.

21 Torre D. Cryosurgery of basal cell carcinoma. 7 Am Acad Dermatol 1986; $15(5): 917-29$.

22 Greenway HT, Cornell RC, Tanner DJ, Peets E, Bordin GM Nagi $L$. Treatment of basal cell carcinoma with intralesional interferon. 7 Am Acad Dermatol 1986; 15 (3): 437-43.

23 Waldow SM, LoBracio RV, Kohler IK, Wallk S, Fritts HT. Photodynamic therapy for treatment of malignant cutaneous lesions. Laser Surg Med 1987; 7: 451-6.

24 Peck GL, DiGiovanna JJ, Sarnoff DS, et al. Treatment and prevention of basal cell carcinoma with oral isotretinoin. F Am Acad Dermatol 1988; 19 (1): 176-85.

25 Ryan RF, Marks MW. Topical 5-fluorouracil in the treatmen of carcinoma of the nasal floor and nasal alae. Ann Plast Surg 1988; 20: 48-54.

26 Reis R, Klastersky J. Nephrotoxicity induced by cancer chemotherapy with special emphasis on cis-platin toxicity.

27 Peil IJ, Myer D, Perlia CP. Effects of cis-diamminedichloroplatinum (II) (NCS-11985) on hearing function in man Cancer Chemother Rep 1974; 58: 871-5.

28 Berman IJ, Mann MP. Seizures and transient cortical blindness associated with cis-platinum (II) diamminedichloride (PDD) therapy in a thirty-year-old man. Cancer 1980; 45 764-6.

29 Pippett CH, Muss HB, Homesley HD, Jobson VW. Cisplatinum associated cortical blindness. Gynecol Oncol 1981; 12: $253-5$.

30 Walsh TJ, Clark AW, Parhad IM, Green WR. Neurotoxic effects of cisplatin therapy. Arch Neurol 1982; 39: 719-20.

31 Becher R, Schutt P, Oseika R, Schmidt CG. Periphera neuropathy and ophthalmic toxicity after treatment with cisneuropathy and ophthalmic toxicity after treatment with cisdich: 219-21.

32 Cohen RJ, Cuneo RA, Cruciger MP, Jackman AE. Transien left homonomous hemianopsia and encephalopathy following treatment of testicular carcinoma with cisplatinum, vinblastine and bleomycin. 7 Clin Oncol 1983; 1: 392-3.

33 Ostrow S, Hahn D, Wiernik PH, Richards RD. Ophthalmic toxicity after cis-dichlorodiammineplatinum (II) therapy. Cancer Treat Rep 1978; 62: 1591-654.

34 Griffin JD, Garnick MB. Eye toxicity of cancer chemotherapy: a review of the literature. Cancer 1981; 48: 1539-49. 